\title{
Rotational thromboelastometry-guided transfusion during lumbar pedicle subtraction osteotomy for adult spinal deformity: preliminary findings from a matched cohort study
}

\author{
Thomas J. Buell, MD, ${ }^{1}$ Davis G. Taylor, MD,, Ching-Jen Chen, MD, ${ }^{1}$ Lauren K. Dunn, MD, PhD, ${ }^{2}$ \\ Jeffrey P. Mullin, MD, ${ }^{3}$ Marcus D. Mazur, MD, ${ }^{4}$ Chun-Po Yen, MD, ${ }^{1}$ Mark E. Shaffrey, MD, ${ }^{1}$ \\ Christopher I. Shaffrey, MD, ${ }^{1}$ Justin S. Smith, MD, PhD, ${ }^{1}$ and Bhiken I. Naik, MBBCh ${ }^{1,2}$
}

Departments of ${ }^{1}$ Neurosurgery and ${ }^{2}$ Anesthesiology, University of Virginia, Charlottesville, Virginia; ${ }^{3}$ Department of Neurosurgery, University of Buffalo, New York; and ${ }^{4}$ Department of Neurosurgery, University of Utah, Salt Lake City, Utah

\begin{abstract}
OBJECTIVE Significant blood loss and coagulopathy are often encountered during adult spinal deformity (ASD) surgery, and the optimal intraoperative transfusion algorithm is debatable. Rotational thromboelastometry (ROTEM), a functional viscoelastometric method for real-time hemostasis testing, may allow early identification of coagulopathy and improve transfusion practices. The objective of this study was to investigate the effect of ROTEM-guided blood product management on perioperative blood loss and transfusion requirements in ASD patients undergoing correction with pedicle subtraction osteotomy (PSO).

METHODS The authors retrospectively reviewed patients with ASD who underwent single-level lumbar PSO at the University of Virginia Health System. All patients who received ROTEM-guided blood product transfusion between 2015 and 2017 were matched in a 1:1 ratio to a historical cohort treated using conventional laboratory testing (control group). Co-primary outcomes were intraoperative estimated blood loss (EBL) and total blood product transfusion volume. Secondary outcomes were perioperative transfusion requirements and postoperative subfascial drain output.
\end{abstract}

RESULTS The matched groups (ROTEM and control) comprised 17 patients each. Comparison of matched group baseline characteristics demonstrated differences in female sex and total intraoperative dose of intravenous tranexamic acid (TXA). Although EBL was comparable between ROTEM versus control $(3200.00 \pm 2106.24 \mathrm{ml}$ vs $3874.12 \pm 2224.22$ $\mathrm{ml}, \mathrm{p}=0.36$ ), there was a small to medium effect size (Cohen's $d=0.31$ ) on EBL reduction with ROTEM. The ROTEM group had less total blood product transfusion volume (1624.18 $\pm 1774.79 \mathrm{ml}$ vs $2810.88 \pm 1847.46 \mathrm{ml}, \mathrm{p}=0.02)$, and the effect size was medium to large (Cohen's $d=0.66$ ). This difference was no longer significant after adjusting for TXA ( $\beta$ $=-0.18,95 \%$ confidence interval $[\mathrm{Cl}]-1995.78$ to $671.64, p=0.32)$. More cryoprecipitate and less fresh frozen plasma (FFP) were transfused in the ROTEM group patients (cryoprecipitate units: $1.24 \pm 1.20$ vs $0.53 \pm 1.01, p=0.03$; FFP volume: $119.76 \pm 230.82 \mathrm{ml}$ vs $673.06 \pm 627.08 \mathrm{ml}, \mathrm{p}<0.01$ ), and this remained significant after adjusting for TXA (cryoprecipitate units: $\beta=0.39,95 \% \mathrm{Cl} 0.05$ to $1.73, p=0.04$; FFP volume: $\beta=-0.41,95 \% \mathrm{Cl}-772.55$ to $-76.30, p=0.02$ ). Drain output was lower in the ROTEM group and remained significant after adjusting for TXA.

CONCLUSIONS For ASD patients treated using lumbar PSO, more cryoprecipitate and less FFP were transfused in the ROTEM group compared to the control group. These preliminary findings suggest ROTEM-guided therapy may allow early identification of hypofibrinogenemia, and aggressive management of this may reduce blood loss and total blood product transfusion volume. Additional prospective studies of larger cohorts are warranted to identify the appropriate subset of ASD patients who may benefit from intraoperative ROTEM analysis.

https://thejns.org/doi/abs/10.3171/2019.1.FOCUS18572

KEYWORDS rotational thromboelastometry; spine surgery; hypofibrinogenemia; bleeding; adult spinal deformity; scoliosis; pedicle subtraction osteotomy

ABBREVIATIONS ASA = American Society of Anesthesiologists; $\mathrm{ASD}=$ adult spinal deformity; $\mathrm{Cl}=$ confidence interval; $\mathrm{EBL}=\mathrm{estimated}$ blood loss; $\mathrm{EXTEM}=\mathrm{external}$ temogram; FFP = fresh frozen plasma; FIBTEM = fibrinogen temogram; ICU = intensive care unit; INR = international normalized ratio; $P O C=$ point-of-care; POD = postoperative day; $\mathrm{pRBC}=$ packed red blood cell; $\mathrm{PSO}=$ pedicle subtraction osteotomy; ROTEM = rotational thromboelastometry; $\mathrm{SPO}=\mathrm{Smith}-\mathrm{Petersen}$ osteotomy; TLIF = transforaminal lumbar interbody fusion; TXA = tranexamic acid.

SUBMITTED October 22, 2018. ACCEPTED January 24, 2019.

INCLUDE WHEN CITING DOI: 10.3171/2019.1.FOCUS18572. 
A DULT spinal deformity (ASD) surgery is associated with significant blood loss, especially when cases involve 3-column osteotomies., , $5,36-38$ Intraoperative blood loss in excess of $4 \mathrm{~L}$ has been reported in as many as $24 \%$ of patients who have surgery involving 3 -column osteotomies. ${ }^{25}$ The need for blood transfusions to compensate for these losses is associated with significant complications such as infections, thromboembolic

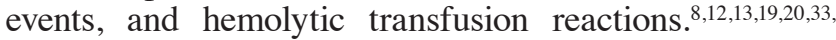
34,39,41,46 Patients requiring blood transfusion have been shown to have prolonged hospitalization, increased readmission and reoperation rates, and higher surgery-related costs. $^{12,22,29,34}$ Because of these various complications, there have been significant efforts to reduce the need for perioperative blood product transfusion. 7,14,16,23,24,28,47

Utilization of rotational thromboelastometry (ROTEM) for rapid intraoperative evaluation of the coagulation system may allow early detection and management of coagulopathy and thereby reduce blood loss and perioperative transfusion requirements. ${ }^{21,24}$ ROTEM is a dynamic functional hemostatic test based on viscoelastic measurement changes of whole blood during clot formation. ${ }^{21,24}$ Unlike standard laboratory tests (thrombin and prothrombin time and activated partial thromboplastin time), ROTEM can detect abnormalities of the different coagulation pathways such as platelet adhesion/aggregation, coagulation, clot strength, and fibrinolysis with much faster turnaround time..$^{21,24}$ The interaction between coagulation factors and cellular components during the coagulation and subsequent lytic phase can be evaluated and reported in real time. ${ }^{21}$ In multiple studies, use of thromboelastometry has been demonstrated to improve profiling of coagulation deficiencies and reduce blood product utilization. However, most of these studies have been restricted to cardiac, trauma, and orthotopic liver transplantation surgery..$^{11,17,27,30-32,35,40,42}$

Few studies suggest ROTEM may have benefits over standard laboratory testing for major spine surgery. ${ }^{14,24}$ However, none have focused on the more complex subset of adult scoliosis cases in which pedicle subtraction osteotomy (PSO) is performed. PSO is a technically challenging technique that is often associated with higher blood loss and morbidity. ${ }^{1,3,5,37}$ The objective of this pilot study was to provide a preliminary assessment of ROTEMguided blood product management for patients with ASD undergoing lumbar PSO for deformity correction. We hypothesized that intraoperative ROTEM analysis may allow early identification of intraoperative coagulation deficiencies, and aggressive management may reduce perioperative blood loss and transfusion requirements.

\section{Methods}

\section{Patient Selection}

This study was approved by the University of Virginia IRB for Health Sciences Research. The requirement for patient informed consent was waived. We performed a retrospective review of a prospectively collected database of patients with ASD (age $\geq 18$ years) who underwent single-level lumbar PSO for deformity correction at our institution. Patients who were managed intraop- eratively using ROTEM-guided therapy between June 2015 and June 2017 were identified as the ROTEM cohort. Using an institutional database, patients who did not receive intraoperative ROTEM guidance were identified between December 2010 and September 2014 as the historical control cohort. Study inclusion criteria can be summarized as follows: 1) anticipated intraoperative blood loss $\geq 1000 \mathrm{ml}$; 2) anticipated PSO at one lumbar level; 3) anticipated need for allogeneic blood products; and 4) anticipated postoperative intensive care unit (ICU) admission.

\section{Data Collection}

Preoperative baseline demographic data included age, sex, BMI, American Society of Anesthesiologists (ASA) Physical Classification System grade, and history of prior lumbar spine fusion. Preoperative laboratory data included hemoglobin level, platelet count, international normalized ratio (INR), and fibrinogen. These laboratory parameters were also recorded postoperatively (typically $\leq 6$ hours after surgery). Surgical data included number of instrumented levels, number of transforaminal lumbar interbody fusions (TLIFs), number of Smith-Petersen osteotomies (SPOs), use of sacropelvic fixation with iliac screws, operative duration, and total dose of intravenous tranexamic acid (TXA).

\section{Outcomes}

Co-primary outcomes were intraoperative estimated blood loss (EBL) and total blood product requirements (packed red blood cells [pRBCs], cryoprecipitate, fresh frozen plasma [FFP], and platelets). Secondary outcomes were pRBCs (allogeneic and autologous), cryoprecipitate, FFP, platelets transfused during surgery, intraoperative crystalloid and colloid transfusion, postoperative subfascial drain output, days to ambulation, days in the ICU, and length of hospitalization.

\section{Transfusion Algorithm}

Our algorithms for intraoperative ROTEM-guided transfusion and point-of-care (POC) or conventional laboratory results-driven blood product management have been previously described in detail. ${ }^{24}$ Briefly, ROTEMguided transfusion comprised functional coagulation assessment performed with external temogram (EXTEM) and fibrinogen temogram (FIBTEM) assays. Intraoperative transfusion for temogram abnormalities was protocol-based and directed by a predefined treatment algorithm (Fig. 1A). Initial ROTEM results were obtained after induction of anesthesia. Then, further intraoperative ROTEM data were obtained after 1-2 units of EBL and at regular intervals at the discretion of the anesthesiologist. Frequency of laboratory testing was at the discretion of the anesthesiologist in POC or conventional laboratory results-driven blood product management (Fig. 1B). In both algorithms, pRBC transfusions were performed to maintain a hemoglobin level $\geq 9 \mathrm{~g} / \mathrm{dl}$.

\section{Intraoperative TXA Administration Protocol}

Emerging evidence suggests efficacy of intraopera- 

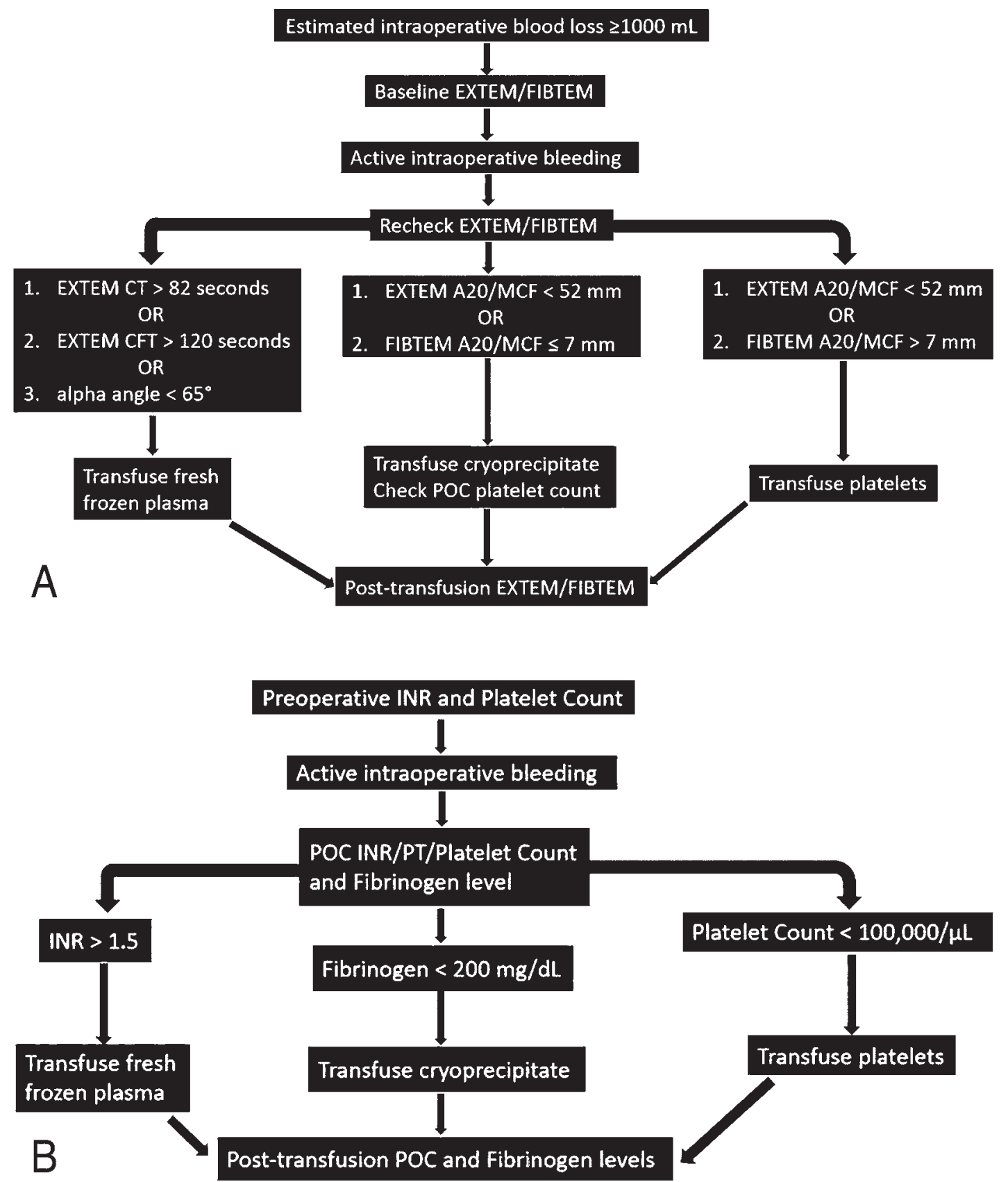

FIG. 1. A: Predefined treatment algorithm for patients receiving intraoperative ROTEM-guided transfusion therapy (recreated from Naik et al.). ${ }^{24}$ ROTEM assessment includes EXTEM and FIBTEM assays. Algorithm variables reported by these assays are clotting time (CT), clot formation time (CFT), alpha angle, amplitude at 20 minutes (A20), and maximum clot firmness (MCF). Prolongation of EXTEM CT (> 82 seconds) is related to extrinsic pathway abnormalities (due to functionally low levels of factors II, VII, and X), which is treated with fresh frozen plasma. ${ }^{43}$ Low EXTEM A20 and MCF $(<52 \mathrm{~mm})$ indicate poor clot strength, which may be related to either platelet abnormalities (when FIBTEM MCF is normal [7-24 mm]; treated with platelet transfusion) or low fibrinogen (when FIBTEM MCF is low $[\leq 7 \mathrm{~mm}]$; treated with cryoprecipitate transfusion). B: Predefined treatment algorithm for patients receiving intraoperative transfusion therapy guided by conventional or POC testing (recreated from Naik et al. ${ }^{24}$ ). POC testing for prothrombin time (PT), INR, and platelet count was performed in the blood bank. Assessment of fibrinogen levels was performed via standard laboratory testing.

tive systemic TXA for reducing blood loss in complex spine surgery. ${ }^{45,47}$ Consequently, our institution has increasingly employed various algorithms for administering TXA during surgery. For this study, all preoperative patients were screened for potential contraindications to intravenous TXA (deep venous thrombosis, pulmonary embolism, stroke, myocardial infarction, cardiac stenting, renal impairment, seizures, or hypercoagulable dis- 


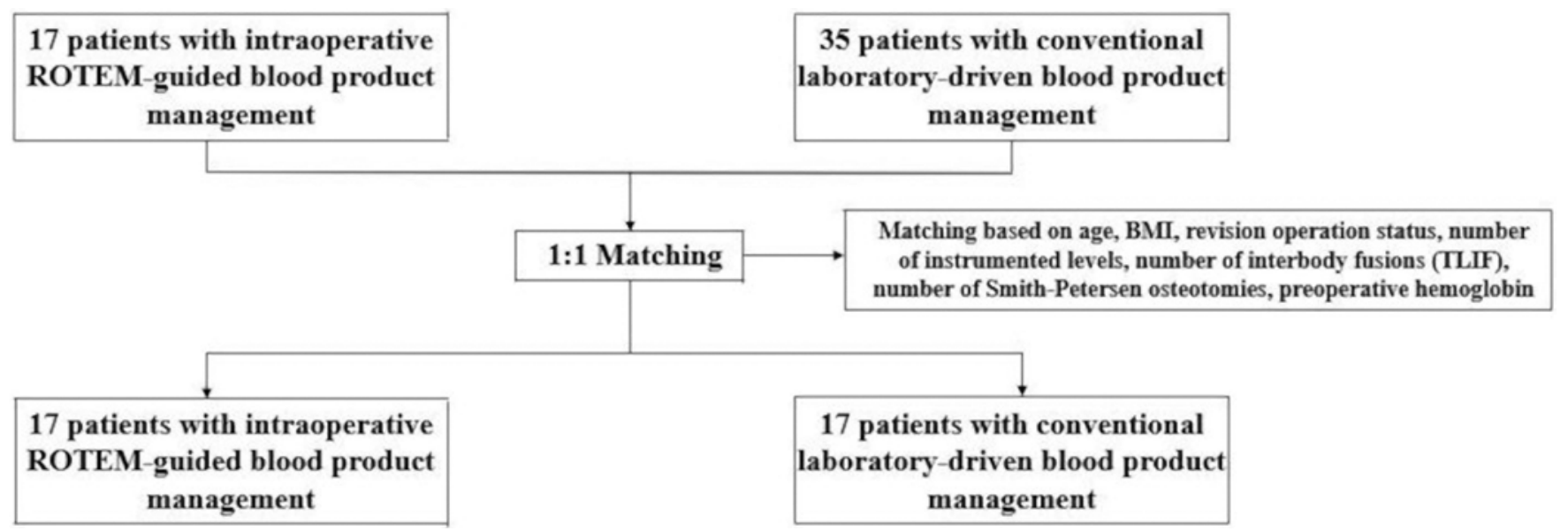

FIG. 2. Flowchart showing the selection process of matched ROTEM and conventional laboratory testing (control) cohorts.

orders). ${ }^{45,47}$ After multidisciplinary discussion (between the attending complex spine surgeon and neuroanesthesiologist), patients who were deemed suitable candidates for intravenous TXA were subject to review of standard hematological laboratory parameters (i.e., platelet count, INR, partial thromboplastin time). After further multidisciplinary assessment, suitable patients were enrolled in our institution's neuroanesthesiology prospective data set for TXA administration. A common intravenous dosing regimen utilized for ROTEM patients was $30 \mathrm{mg} / \mathrm{kg}$ loading bolus followed by $5 \mathrm{mg} / \mathrm{kg} / \mathrm{hr}$ continuous infusion (unless there was a history of thromboembolic events). Lower doses were administered to patients with relative contraindications to systemic TXA. Historically, the thrombotic complication profile of intraoperative TXA administration was unclear; ${ }^{45}$ therefore, common intravenous doses for control patients were generally lower $(10 \mathrm{mg} / \mathrm{kg}$ loading bolus followed by $1 \mathrm{mg} / \mathrm{kg} / \mathrm{hr}$ continuous infusion). As discussed below, the potential confounding effect of a coagulation-modifying agent such as TXA on assessed study outcomes was controlled using multiple linear regression.

\section{Statistical Analysis}

Statistical analyses were performed using SPSS (version 24.0, IBM Corp.) and Stata (version 14.2, StataCorp). Patients who received intraoperative ROTEM-guided therapy (ROTEM cohort) were matched to patients in the historical control cohort treated using the POC or conventional laboratory approach (control cohort). Matching was performed in a 1:1 ratio with a caliper of 0.25 , using propensity scores derived from baseline demographic, laboratory, and surgical covariates (age, BMI, revision operation status, number of instrumented levels, number of TLIFs, number of SPOs, and preoperative hemoglobin). In rare cases, blood product transfusion units were recorded without specifying volume; therefore, missing volumes were imputed from volume per unit averages of the entire data set.

Primary and secondary outcomes between the matched groups were then compared. Normality of data in each matched group was assessed using the Shapiro-Wilk test.
Continuous variables were compared using the Student's t-test or Mann-Whitney U-test, as appropriate. Categorical variables were compared using Pearson's chi-square or Fisher's exact tests, as appropriate. These outcomes were then adjusted for residual baseline differences after the matching process using multiple linear regression. Statistical significance was defined as $p<0.05$, and all tests were 2-tailed. Due to small sample size, we computed a Cohen's d-based effect size estimation to compare the quantitative strength of ROTEM-based transfusion on primary outcomes. ${ }^{9}$

\section{Results \\ Patient Cohort}

Intraoperative ROTEM-guided blood product management was utilized during 17 ASD operations that involved single-level lumbar PSO between 2015 and 2017. After performing propensity-score matching using a historical database of patients with PSO who had conventional laboratory testing, the matched cohorts (ROTEM and control) comprised 17 patients each (Fig. 2).

\section{Comparison of Baseline Characteristics of Matched Groups}

Table 1 compares the baseline characteristics between the matched groups. Between the matched groups, female sex $(94 \%$ vs $59 \%, p=0.04)$ was more frequent and total intravenous TXA administered $(3534.38 \pm 2443.90 \mathrm{mg}$ vs $1916.93 \pm 1862.77 \mathrm{mg}, \mathrm{p}=0.03$ ) was higher in the ROTEM group compared to the control group. Table 2 compares preoperative and initial postoperative laboratory results between matched cohorts. Preoperative and initial postoperative hemoglobin, platelet count, INR, and fibrinogen were comparable between matched cohorts.

\section{Comparison of Co-Primary Outcomes Between Matched Groups}

Table 3 compares the co-primary outcomes between the matched groups. ROTEM patients had less EBL compared to controls, but the difference did not reach significance $(3200.00 \pm 2106.24$ vs $3874.12 \pm 2224.22 \mathrm{ml}, \mathrm{p}=$ 
TABLE 1. Preoperative baseline demographic and surgical parameters

\begin{tabular}{lccc}
\hline \multicolumn{1}{c}{ Variable } & ROTEM $(\mathrm{n}=17)$ & Conventional $(\mathrm{n}=17)$ & $\mathrm{p}$ Value \\
\hline Mean age at surgery $\pm \mathrm{SD}$, yrs & $62.94 \pm 11.58$ & $64.29 \pm 11.80$ & 0.62 \\
\hline Female, $\mathrm{n}(\%)$ & $16(94)$ & $10(59)$ & 0.04 \\
\hline Mean BMI \pm SD, $\mathrm{kg} / \mathrm{m}^{2}$ & $30.14 \pm 7.68$ & $30.22 \pm 4.49$ & 0.27 \\
\hline Median ASA physical status score (range) & $3(2-3)$ & $3(2-3)$ & 1.00 \\
\hline Revision operations, $\mathrm{n}(\%)$ & $16(94)$ & $16(94)$ & 1.00 \\
\hline Mean no. of instrumented levels \pm SD & $11.47 \pm 3.10$ & $12.47 \pm 3.45$ & 0.67 \\
\hline Mean no. of TLIFs \pm SD & $0.59 \pm 0.80$ & $0.53 \pm 0.62$ & 1.00 \\
\hline Mean no. of SPOs \pm SD & $1.47 \pm 1.87$ & $1.71 \pm 2.39$ & 0.96 \\
\hline liac fixation, $\mathrm{n}(\%)$ & $16(94)$ & $16(94)$ & 1.00 \\
\hline Mean operative duration $\pm \mathrm{SD}$, min & $382.71 \pm 80.15$ & $382.94 \pm 67.85$ & 0.59 \\
\hline Mean TXA \pm SD, $\mathrm{mg}$ & $3534.38 \pm 2443.90$ & $1916.93 \pm 1862.77$ & 0.03 \\
\hline
\end{tabular}

Boldface type indicates statistical significance.

0.36). This difference remained nonsignificant after adjusting for TXA $(\beta=-0.07,95 \%$ confidence interval $[\mathrm{CI}]$ -1858.67 to $1330.13, \mathrm{p}=0.74)$. Total blood product transfusion volume was lower in the ROTEM group compared to controls $(1624.18 \pm 1774.79$ vs $2810.88 \pm 1847.46 \mathrm{ml}$, $\mathrm{p}=0.02)$. This difference was no longer significant after adjusting for TXA $(\beta=-0.18,95 \% \mathrm{CI}-1995.78$ to 671.64 , $\mathrm{p}=0.32$ ).

Effect sizes were small to medium (Cohen's $d=0.31$ ) and medium to large (Cohen's $\mathrm{d}=0.66$ ) for differences in EBL and total blood product transfusion volume, respectively, between matched groups.

\section{Comparison of Secondary Outcomes Between Matched Groups}

Table 4 compares the secondary intraoperative outcomes between the matched groups. Fewer units of pRBCs were transfused in the ROTEM group compared to the control group $(3.24 \pm 2.68$ vs $5.29 \pm 3.3, \mathrm{p}=0.048)$. This difference was no longer significant after adjusting for TXA administration $(\beta=-0.88,95 \%$ CI -3.03 to 1.21 , $\mathrm{p}=0.39$ ). The transfused $\mathrm{pRBC}$ volumes were comparable between the matched groups and remained nonsignificant after adjusting for TXA. The volumes of transfused autologous blood were also comparable between the matched groups and remained nonsignificant after adjusting for TXA.

More units of cryoprecipitate were transfused in the ROTEM group compared to the control group $(1.24 \pm 1.20$ vs $0.53 \pm 1.01, p=0.03)$. This remained significant after adjusting for TXA ( $\beta=0.39,95 \%$ CI 0.05 to $1.73, p=$ 0.04 ). Volume of cryoprecipitate transfused was higher in the ROTEM group compared to controls $(163.88 \pm 167.17$ $\mathrm{ml}$ vs $78.41 \pm 149.61 \mathrm{ml}, \mathrm{p}=0.04)$. However, this difference was no longer significant after adjusting for TXA ( $\beta$ $=0.34,95 \% \mathrm{CI}-13.18$ to $229.04, \mathrm{p}=0.08$ ).

Fewer units of FFP were transfused in the ROTEM group compared to the control group $(1.29 \pm 3.65$ vs $3.94 \pm$ $3.29, \mathrm{p}<0.01)$. However, this difference was no longer significant after adjusting for TXA $(\beta=-0.30,95 \% \mathrm{CI}-4.92$ to $0.48, \mathrm{p}=0.10)$. Volume of FFP transfused was lower in the ROTEM group compared to controls $(119.76 \pm 230.82 \mathrm{ml}$ vs $673.06 \pm 627.08 \mathrm{ml}, \mathrm{p}<0.01)$. This difference remained significant after adjusting for TXA $(\beta=-0.41,95 \%$ CI -772.55 to $-76.30, \mathrm{p}=0.02)$.

Platelet transfusions (platelet units and volume) were comparable between the matched groups. These remained nonsignificant after adjusting for TXA. Volumes of crystalloid infused were comparable between the matched groups and remained nonsignificant after adjusting for TXA. Volume of colloid infused was higher in the ROTEM group compared to the control group (2329.41 $\pm 1217.84 \mathrm{ml}$ vs $1411.76 \pm 744 \mathrm{ml}, \mathrm{p}=0.01)$, and this remained significant after adjusting for TXA $(\beta=0.37,95 \%$ CI 23.86 to $1598.08, \mathrm{p}=0.04$ ).

Table 5 compares the secondary postoperative outcomes between the matched groups. Postoperative subfascial drain output was reduced in the ROTEM cohort after postoperative day 1 (POD1). After controlling for TXA, drain output remained significantly reduced in the ROTEM cohort after POD2 (Fig. 3). Total postoperative volumes of blood product transfused were comparable between the two groups. Time to ambulation, length of ICU stay, and time to discharge from hospital were also comparable between the matched groups.

TABLE 2. Comparison of perioperative lab results

\begin{tabular}{lccc}
\hline Laboratory Results & ROTEM & Conventional & p Value \\
\hline Preop & & & \\
\hline Hemoglobin $(\mathrm{g} / \mathrm{dl})$ & $12.46 \pm 0.90$ & $12.89 \pm 1.70$ & 0.37 \\
\hline Platelet count $(/ \mu \mathrm{l})$ & $251.87 \pm 55.19$ & $231.53 \pm 62.64$ & 0.34 \\
\hline INR & $1.00 \pm 0.12$ & $1.01 \pm 0.09$ & 0.42 \\
\hline Fibrinogen $(\mathrm{mg} / \mathrm{dl})$ & $336.31 \pm 44.53$ & $363.09 \pm 78.98$ & 0.31 \\
\hline Initial postop & & & \\
\hline Hemoglobin $(\mathrm{g} / \mathrm{dl})$ & $9.75 \pm 1.23$ & $10.27 \pm 1.02$ & 0.19 \\
\hline Platelet count $(/ \mu \mathrm{l})$ & $108.56 \pm 31.08$ & $110.94 \pm 38.59$ & 0.85 \\
\hline INR & $1.24 \pm 0.15$ & $1.23 \pm 0.13$ & 0.72 \\
\hline Fibrinogen $(\mathrm{mg} / \mathrm{dl})$ & $175.13 \pm 47.44$ & $181.83 \pm 66.44$ & 0.80 \\
\hline
\end{tabular}

Values are expressed as mean \pm SD unless otherwise indicated. 
TABLE 3. Primary outcomes of intraoperative EBL and total blood product transfusion requirements

\begin{tabular}{lcccccc}
\hline \multicolumn{1}{c}{ Variable } & ROTEM & Conventional & $p$ Value & Cohen's d & $\beta(95 \%$ Cl) & Adjusted $p$ Value* \\
\hline EBL $(\mathrm{ml})$ & $3200.00 \pm 2106.24$ & $3874.12 \pm 2224.22$ & 0.36 & 0.31 & $-0.07(-1858.67$ to 1330.13) & 0.74 \\
\hline $\begin{array}{c}\text { Total blood product transfusion } \\
\text { volume }(\mathrm{ml})\end{array}$ & $1624.18 \pm 1774.79$ & $2810.88 \pm 1847.46$ & 0.02 & 0.66 & $-0.18(-1995.78$ to 671.64$)$ & 0.32 \\
\hline
\end{tabular}

Values expressed as mean \pm SD unless otherwise indicated. Boldface type indicates statistical significance.

* Values were adjusted for intraoperative administration of intravenous TXA.

\section{Discussion}

Surgical management of ASD is often associated with high blood loss and transfusion requirements. ${ }^{2,4,5,25,36-38}$ The significant blood loss and transfusions may be related to several factors, such as the relatively advanced age of many patients with ASD, their high rate of associated comorbidities, as well as surgically related factors (i.e., posterior approach, pelvic fixation, osteotomy, and longer operative duration). ${ }^{26,42}$ The need for blood transfusions to compensate for these losses is associated with perioperative complications such as infections, thromboembolic events, and hemolytic transfusion reactions. , $^{82,13,19,20,33,34,39,41,46}$ Also, patients requiring blood transfusion have been shown to have prolonged hospitalization, increased readmission and reoperation rates, and higher surgery-related costs. ${ }^{12,22,29,34}$ Because of these various complications, there have been significant efforts to reduce the need for perioperative blood product transfusions. $7,14,16,23,24,28,47$

ROTEM analysis may have novel applications in ASD surgery if it allows real-time hematological assessment for rapid correction of intraoperative coagulopathies. ${ }^{14,24}$ The utility of ROTEM-guided blood product management has previously been demonstrated in cardiac, trauma, and

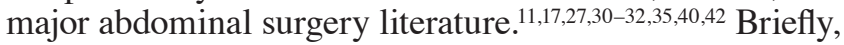
ROTEM measures the viscoelastic properties of whole blood. ${ }^{21}$ First, a rotating pin is inserted into a cuvette containing citrated whole blood. ${ }^{21}$ There is a $1-\mathrm{mm}$ distance between the pin and cuvette, which is bridged by the blood. ${ }^{24}$ ROTEM analysis and clot formation begin when calcium and tissue factor are added to mimic the extrin- sic coagulation pathway (EXTEM). ${ }^{21,24}$ Initially, there is minimal resistance to pin rotation; however, with increasing clot formation there is increased resistance to rotation, which is detected by an optical sensor. This resistance to rotation is reported in real time as both a functional parameter and a representative curve. ${ }^{24}$ In our study, cytochalasin D, a platelet inhibitor, was added to make the results depend on fibrin formation and polymerization alone (FIBTEM). ${ }^{24}$ Our ROTEM-based algorithm for blood product transfusion utilized in this study is summarized in Fig. 1A.

There are limited data on the utility of ROTEM in minimizing blood loss and reducing transfusion requirements in ASD surgery. ${ }^{6,14,24}$ In the first major study on ROTEM and ASD, Naik et al. demonstrated that cryoprecipitate use increased and FFP use decreased in response to ROTEM analysis. ${ }^{24}$ These results suggested that ROTEM analysis was able to identify hypofibrinogenemia earlier than conventional lab testing. ${ }^{24}$ In patients without TXA administration, Naik et al. also demonstrated that ROTEM analysis could significantly reduce blood loss with a trend toward less pRBC transfusion..$^{24}$ In patients with intraoperative TXA administration, there was a nonsignificant reduction in blood loss; however, there was still significantly less FFP and increased cryoprecipitate use in the ROTEM group. ${ }^{24}$ In a separate study comparing ROTEM to conventional laboratory testing, Guan et al. demonstrated that ROTEM patients required significantly less total blood products during their hospitalization compared to patients managed with conventional lab testing..${ }^{14}$ Comparison of these prior ROTEM spine studies is limited by differences

TABLE 4. Secondary intraoperative outcomes compared between the matched cohorts

\begin{tabular}{|c|c|c|c|c|c|}
\hline Variable & ROTEM & Conventional & p Value & $\beta(95 \% \mathrm{Cl})^{*}$ & p Value* \\
\hline pRBC transfusion (units) & $3.24 \pm 2.68$ & $5.29 \pm 3.37$ & 0.05 & $-0.88(-3.03$ to 1.21$)$ & 0.39 \\
\hline pRBC volume (ml) & $1023.59 \pm 849.84$ & $1575.41 \pm 971.26$ & 0.09 & $-0.12(-861.14$ to 422.63$)$ & 0.49 \\
\hline Autologous blood transfusion (ml) & $1148.76 \pm 890.51$ & $1173.76 \pm 712.15$ & 0.67 & $0.07(-510.72$ to 721.20$)$ & 0.73 \\
\hline Cryoprecipitate transfusion (units) & $1.24 \pm 1.20$ & $0.53 \pm 1.01$ & 0.03 & 0.39 (0.05 to 1.73$)$ & 0.04 \\
\hline Cryoprecipitate volume (ml) & $163.88 \pm 167.17$ & $78.41 \pm 149.61$ & 0.04 & $0.34(-13.18$ to 229.04$)$ & 0.08 \\
\hline FFP transfusion (units) & $1.29 \pm 3.65$ & $3.94 \pm 3.29$ & $<0.01$ & $-0.30(-4.92$ to 0.48$)$ & 0.10 \\
\hline FFP volume (ml) & $119.76 \pm 230.82$ & $673.06 \pm 627.08$ & $<0.01$ & $-0.41(-772.55$ to -76.30$)$ & 0.02 \\
\hline Platelet (units) & $0.47 \pm 0.87$ & $0.53 \pm 0.80$ & 0.72 & $0.10(-0.47$ to 0.79$)$ & 0.61 \\
\hline Platelet volume (ml) & $105.18 \pm 196.69$ & $121.24 \pm 184.18$ & 0.73 & $0.08(-113.58$ to 173.12$)$ & 0.67 \\
\hline Crystalloid (ml) & $3070.59 \pm 1079.36$ & $3098.76 \pm 1227.84$ & 0.94 & $0.01(-890.97$ to 918.47$)$ & 0.98 \\
\hline Colloid (ml) & $2329.41 \pm 1217.84$ & $1411.76 \pm 744.46$ & 0.01 & $0.37(23.86$ to 1598.08$)$ & 0.04 \\
\hline
\end{tabular}

Values expressed as mean \pm SD unless otherwise indicated. Boldface type indicates statistical significance.

* Values were adjusted for intraoperative administration of intravenous TXA. 
TABLE 5. Secondary postoperative outcomes compared between the matched cohorts

\begin{tabular}{|c|c|c|c|c|c|}
\hline Variable & ROTEM & Conventional & p Value & $\beta(95 \% \mathrm{Cl})^{*}$ & Adjusted $p$ Value ${ }^{*}$ \\
\hline \multicolumn{6}{|l|}{ Drain output (ml) } \\
\hline POD1 & $491.54 \pm 210.19$ & $564.59 \pm 215.00$ & 0.37 & $-0.12(-225.92$ to 128.74$)$ & 0.58 \\
\hline POD2 & $170.92 \pm 160.20$ & $313.28 \pm 156.55$ & 0.02 & $-0.34(-236.34$ to 14.57$)$ & 0.08 \\
\hline POD3 & $71.42 \pm 83.19$ & $180.00 \pm 116.91$ & 0.01 & $-0.43(-171.17$ to -10.47$)$ & 0.03 \\
\hline POD4 & $22.85 \pm 47.87$ & $111.44 \pm 95.98$ & $<0.01$ & $-0.47(-141.58$ to -14.97$)$ & 0.02 \\
\hline Total blood product transfusion volume (ml) & $923.00 \pm 414.90$ & $1147.82 \pm 896.67$ & 0.37 & $-0.11(-732.65$ to 435.29$)$ & 0.61 \\
\hline pRBC (units) & $2.31 \pm 1.38$ & $2.18 \pm 1.55$ & 0.81 & $-0.05(-1.33$ to 1.05$)$ & 0.81 \\
\hline pRBC volume $(\mathrm{ml})$ & $714.00 \pm 427.16$ & $678.18 \pm 476.83$ & 0.83 & $-0.04(-407.59$ to 332.59$)$ & 0.84 \\
\hline Cryoprecipitate (units) & $0.23 \pm 0.44$ & $0.18 \pm 0.53$ & 0.77 & $0.06(-0.35$ to 0.47$)$ & 0.77 \\
\hline Cryoprecipitate volume (ml) & $27.85 \pm 53.65$ & $23.41 \pm 71.21$ & 0.85 & $0.06(-48.31$ to 59.05$)$ & 0.84 \\
\hline FFP (units) & $0.15 \pm 0.38$ & $0.65 \pm 1.17$ & 0.12 & $-0.12(-0.68$ to 0.36$)$ & 0.53 \\
\hline FFP volume (ml) & $44.31 \pm 108.71$ & $187.29 \pm 327.89$ & 0.11 & $-0.12(-192.19$ to 99.53$)$ & 0.52 \\
\hline Platelet (units) & $0.54 \pm 0.66$ & $1.12 \pm 1.27$ & 0.12 & $-0.17(-1.20$ to 0.49$)$ & 0.40 \\
\hline Platelet volume (ml) & $136.85 \pm 166.33$ & $258.94 \pm 285.56$ & 0.15 & $-0.15(-263.81$ to 123.39$)$ & 0.46 \\
\hline Days to ambulation & $2.82 \pm 1.94$ & $2.54 \pm 1.20$ & 0.67 & - & - \\
\hline Days in ICU stay & $3.00 \pm 1.41$ & $2.36 \pm 1.01$ & 0.18 & - & - \\
\hline Days to discharge & $6.92 \pm 2.02$ & $7.94 \pm 2.05$ & 0.19 & - & - \\
\hline
\end{tabular}

Values expressed as mean \pm SD unless otherwise indicated; 4 ROTEM patients removed due to postop TXA usage. Boldface type indicates statistical significance.

* Values were adjusted for intraoperative administration of intravenous TXA.

in ROTEM-transfusion algorithms; most notably, the Guan et al. study algorithm did not administer cryoprecipitate. ${ }^{6,14}$

With a significant increase in the number and complexity of ASD corrective surgeries, blood loss often requiring massive intraoperative transfusions becomes a major limiting factor during surgery. ${ }^{15,37}$ This scenario is particularly important during 3-column osteotomies such as the PSO, where extensive intraoperative blood loss may pose a major risk to the patient and prevent execution of the pro-

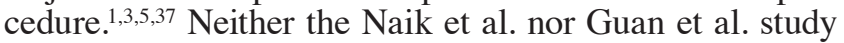
focused on this more complex, morbid subset of ASD surgery with PSO. ${ }^{14,24}$ Therefore, our study objective was to investigate the effect of ROTEM-guided blood product management on perioperative blood loss and transfusion requirements in patients with ASD undergoing lumbar PSO (often for rigid deformity in revision cases). The preliminary findings of our pilot study are consistent with the prior Naik et al. study. First, cryoprecipitate use was increased and FFP use decreased in response to ROTEM analysis, which we hypothesize is a result of earlier identification of hypofibrinogenemia as a major contributor to ongoing coagulopathy in PSO cases. Next, our results demonstrated a small to medium effect size (Cohen's $\mathrm{d}=$ 0.31) on EBL reduction associated with ROTEM analysis. Although there was less EBL in the ROTEM group, this difference was not significant. This lack of significance may be because this pilot retrospective study was likely underpowered, and we hypothesize that further prospec-

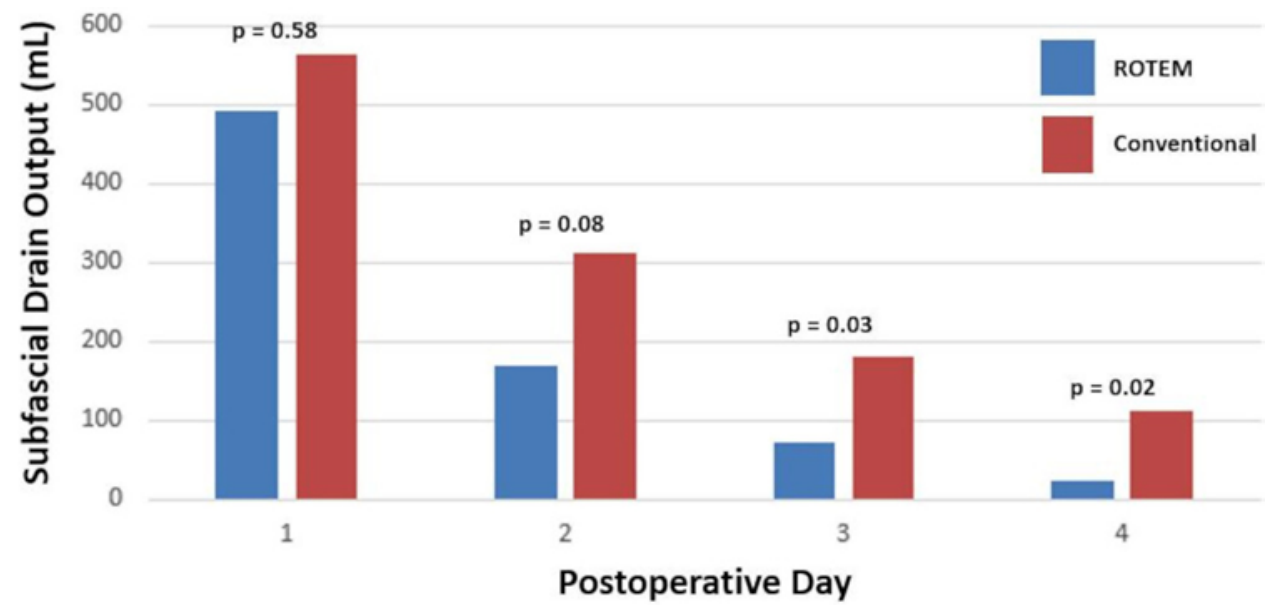

FIG. 3. Postoperative subfascial drain output was significantly reduced in the ROTEM cohort after POD1. After controlling for TXA administration, drain output remained significantly reduced in the ROTEM cohort after POD2. 
tive studies with larger cohorts are necessary to produce more definitive results.

The preliminary findings of this pilot study demonstrated that ROTEM analysis was associated with a significant reduction in total blood product transfusion volume. This should be interpreted with caution because this may be confounded by intraoperative TXA administration. Of note, the effect size of ROTEM analysis on total blood product transfusion volume was larger compared to its effect on blood loss. Based on Cohen's d analysis, the effect size for total blood product transfusion between matched cohorts was 0.66 , indicating a medium to large effect.

We also sought to determine if ROTEM analysis could have an effect on clinical outcomes. There were no significant differences based on days to ambulation, ICU stay, and length of hospitalization. Again, we believe a larger study cohort is likely needed to demonstrate a significant effect on these clinical outcome parameters. However, our analysis did demonstrate a significant reduction in postoperative subfascial drain output by POD2. This suggests ROTEM analysis may lead to earlier removal of surgical drains and less discomfort for patients during convalescence. ${ }^{18}$ Also, this result may suggest ROTEM analysis was indeed beneficial by allowing earlier correction of surgery-related coagulopathy (hypofibrinogenemia), and that the significant effects of blood loss reduction in our study may have been detected postoperatively (by analyzing drain output) rather than intraoperatively with EBL assessment.

It is important to recognize the limitations of the current study. The retrospective, single-center study design utilized in our analysis has inherent limitations. Our results are susceptible to the inherent selection, treatment, and referral biases of our institution and physicians. Also, despite attempts to adjust for baseline differences using multivariable models and propensity-score matching, we acknowledge that a prospective, randomized study would have been a more rigorous and less biased design. It is also important to recognize that a higher total intraoperative dose of TXA was administered to ROTEM patients in this study. This may be attributable to surgeon preference and possible dose-dependent effects. TXA is a coagulation-modifying drug and may confound comparisons of ROTEM versus conventional laboratory testing for blood loss and transfusion reduction. ${ }^{45}$ Utilization of systemic TXA (and even topical TXA ${ }^{10}$ ) is becoming increasingly common in complex spine surgery. ${ }^{45,47}$ Given the high expected blood loss associated with complex spine operations, $, 5,36,37$ it may not be appropriate to design a study withholding TXA until further evidence suggests higher rates of adverse thromboembolic events. ${ }^{45,47}$ Therefore, to control for TXA as a potential confounder, we utilized multiple linear regression when assessing primary and secondary outcomes in our study. However, we acknowledge the potential for nonlinear variability in the pharmacological dose response between patients that would limit this study. Next, because nonsignificant differences in our primary outcomes (after controlling for TXA) may be due to the relatively small study size, we computed a Cohen's d-based effect size estimation to compare the quantitative strength of ROTEM-based transfusion on EBL and total blood product transfusion volume. ROTEM analysis had a small to medium effect size (Cohen's $d=0.31$ ) on EBL and medium to large effect size (Cohen's $d=0.66$ ) on total blood product transfusion volume. These results may support the notion that this pilot retrospective study was likely underpowered, and that small cohort size may account for the lack of statistically significant differences in some assessed outcomes. However, we think our preliminary findings may still benefit other complex spine surgeons and may serve as a baseline to power future, larger prospective ROTEM trials. Finally, this study is limited to a select subset of patients with ASD undergoing a relatively morbid procedure (i.e., lumbar PSO). Surgeries with PSO have high blood loss from bone bleeding, which is further exacerbated in our ASD cohort because of the relatively advanced age of many patients and thin periosteum with wide vascular channels encountered in osteoporotic bone. ${ }^{5,37}$ Although we intended our analysis to focus on this complex ASD subset, our results may not be generalized to less complex, less morbid spine procedures.

\section{Conclusions}

For patients with ASD treated with lumbar PSO, more cryoprecipitate and less FFP were transfused in the ROTEM group compared to a control group that utilized conventional lab testing to guide blood product transfusion. Our preliminary findings suggest that ROTEM-guided therapy may allow early identification of hypofibrinogenemia (a major contributor to ongoing coagulopathy), and aggressive management of this may reduce blood loss and total blood product transfusion volume. Future prospective studies with larger cohorts are necessary to identify the appropriate subset of patients with ASD who may benefit from intraoperative ROTEM analysis.

\section{References}

1. Bridwell KH, Lewis SJ, Lenke LG, Baldus C, Blanke K: Pedicle subtraction osteotomy for the treatment of fixed sagittal imbalance. J Bone Joint Surg Am 85-A:454-463, 2003

2. Buell TJ, Buchholz AL, Quinn JC, Bess S, Line BG, Ames $\mathrm{CP}$, et al: A pilot study on posterior polyethylene tethers to prevent proximal junctional kyphosis after multilevel spinal instrumentation for adult spinal deformity. Oper Neurosurg (Hagerstown) 16:256-266, 2019

3. Buell TJ, Buchholz AL, Quinn JC, Mullin JP, Garces J, Mazur MD, et al: Extended asymmetrical pedicle subtraction osteotomy for adult spinal deformity: 2-dimensional operative video. Oper Neurosurg (Hagerstown) 16:52-53, 2019

4. Buell TJ, Chen CJ, Quinn JC, Buchholz AL, Mazur MD, Mullin JP, et al: Alignment risk factors for proximal junctional kyphosis and the effect of lower thoracic junctional tethers for adult spinal deformity. World Neurosurg 121:e96-e103, 2019

5. Buell TJ, Nguyen JH, Mazur MD, Mullin JP, Garces J, Taylor DG, et al: Radiographic outcome and complications after single-level lumbar extended pedicle subtraction osteotomy for fixed sagittal malalignment: a retrospective analysis of 55 adult spinal deformity patients with a minimum 2-year follow-up. J Neurosurg Spine 30:242-252, 2018

6. Buell TJ, Taylor DG, Chen CJ, Naik BI: Rotational thromboelastometry-guided transfusion protocol. J Neurosurg Spine 29:118-120, 2018 (Letter)

7. Cheriyan T, Maier SP II, Bianco K, Slobodyanyuk K, Rat- 
tenni RN, Lafage V, et al: Efficacy of tranexamic acid on surgical bleeding in spine surgery: a meta-analysis. Spine J 15:752-761, 2015

8. Cloney M, Dhillon ES, Roberts H, Smith ZA, Koski TR, Dahdaleh NS: Predictors of readmissions and reoperations related to venous thromboembolic events after spine surgery: a single-institution experience with 6869 patients. World Neurosurg 111:e91-e97, 2018

9. Cohen J: A power primer. Psychol Bull 112:155-159, 1992

10. Desai BD, Taylor DG, Chen CJ, Buell TJ, Mullin JP, Naik BI, et al: Utility of topical tranexamic acid for adult patients with spinal deformity and contraindications to systemic tranexamic acid: initial experience and report of 2 cases. J Neurosurg Spine [epub ahead of print January 25, 2019; DOI: $10.3171 / 2018.9 . S P I N E 18204]$

11. Dirkmann D, Görlinger K, Dusse F, Kottenberg E, Peters J: Early thromboelastometric variables reliably predict maximum clot firmness in patients undergoing cardiac surgery: a step towards earlier decision making. Acta Anaesthesiol Scand 57:594-603, 2013

12. Elsamadicy AA, Adogwa O, Vuong VD, Mehta AI, Vasquez RA, Cheng J, et al: Association of intraoperative blood transfusions on postoperative complications, 30-day readmission rates, and 1-year patient-reported outcomes. Spine (Phila Pa 1976) 42:610-615, 2017

13. Fisahn C, Jeyamohan S, Norvell DC, Tubbs RS, Moisi M, Chapman JR, et al: Association between allogeneic blood transfusion and postoperative infection in major spine surgery. Clin Spine Surg 30:E988-E992, 2017

14. Guan J, Cole CD, Schmidt MH, Dailey AT: Utility of intraoperative rotational thromboelastometry in thoracolumbar deformity surgery. J Neurosurg Spine 27:528-533, 2017

15. Gum JL, Carreon LY, Buchowski JM, Lenke LG, Glassman SD: Utilization trends of pedicle subtraction osteotomies compared to posterior spinal fusion for deformity: a national database analysis between 2008-2011. Scoliosis Spinal Disord 11:25, 2016

16. Halanski MA, Cassidy JA, Hetzel S, Reischmann D, Hassan $\mathrm{N}$ : The efficacy of amicar versus tranexamic acid in pediatric spinal deformity surgery: a prospective, randomized, doubleblinded pilot study. Spine Deform 2:191-197, 2014

17. Harr JN, Moore EE, Chin TL, Chapman MP, Ghasabyan A, Stringham JR, et al: Viscoelastic hemostatic fibrinogen assays detect fibrinolysis early. Eur J Trauma Emerg Surg 41:49-56, 2015

18. Hung PI, Chang MC, Chou PH, Lin HH, Wang ST, Liu CL: Is a drain tube necessary for minimally invasive lumbar spine fusion surgery? Eur Spine J 26:733-737, 2017

19. Janssen SJ, Braun Y, Wood KB, Cha TD, Schwab JH: Allogeneic blood transfusions and postoperative infections after lumbar spine surgery. Spine J 15:901-909, 2015

20. Kato S, Chikuda H, Ohya J, Oichi T, Matsui H, Fushimi K, et al: Risk of infectious complications associated with blood transfusion in elective spinal surgery-a propensity score matched analysis. Spine J 16:55-60, 2016

21. Keene DD, Nordmann GR, Woolley T: Rotational thromboelastometry-guided trauma resuscitation. Curr Opin Crit Care 19:605-612, 2013

22. Khanna R, Harris DA, McDevitt JL, Fessler RG, Carabini LM, Lam SK, et al: Impact of anemia and transfusion on readmission and length of stay after spinal surgery: a singlecenter study of 1187 operations. Clin Spine Surg 30:E1338E1342, 2017

23. Lin JD, Lenke LG, Shillingford JN, Laratta JL, Tan LA, Fischer CR, et al: Safety of a high-dose tranexamic acid protocol in complex adult spinal deformity: analysis of 100 consecutive cases. Spine Deform 6:189-194, 2018

24. Naik BI, Pajewski TN, Bogdonoff DI, Zuo Z, Clark P, Terkawi AS, et al: Rotational thromboelastometry-guided blood product management in major spine surgery. J Neurosurg Spine 23:239-249, 2015

25. Norton RP, Bianco K, Lafage V, Schwab FJ: Complications and intercenter variability of three-column resection osteotomies for spinal deformity surgery: a retrospective review of 423 patients. Evid Based Spine Care J 4:157-159, 2013

26. Nguyen JH, Buell TJ, Wang TR, Mullin JP, Mazur MD, Garces J, et al: Low rates of complications after spinopelvic fixation with iliac screws in 260 adult patients with a minimum 2-year follow-up. J Neurosurg Spine [epub ahead of print February 1, 2019; DOI: 10.3171/2018.9.SPINE18239]

27. Ogawa S, Szlam F, Chen EP, Nishimura T, Kim H, Roback JD, et al: A comparative evaluation of rotation thromboelastometry and standard coagulation tests in hemodilutioninduced coagulation changes after cardiac surgery. Transfusion 52:14-22, 2012

28. Peters A, Verma K, Slobodyanyuk K, Cheriyan T, Hoelscher $\mathrm{C}$, Schwab F, et al: Antifibrinolytics reduce blood loss in adult spinal deformity surgery: a prospective, randomized controlled trial. Spine (Phila Pa 1976) 40:E443-E449, 2015

29. Piper K, DeAndrea-Lazarus I, Algattas H, Kimmell KT, Towner J, Li YM, et al: Risk factors associated with readmission and reoperation in patients undergoing spine surgery. World Neurosurg 110:e627-e635, 2018

30. Roullet S, Freyburger G, Cruc M, Quinart A, Stecken L, Audy M, et al: Management of bleeding and transfusion during liver transplantation before and after the introduction of a rotational thromboelastometry-based algorithm. Liver Transpl 21:169-179, 2015

31. Rugeri L, Levrat A, David JS, Delecroix E, Floccard B, Gros A, et al: Diagnosis of early coagulation abnormalities in trauma patients by rotation thrombelastography. J Thromb Haemost 5:289-295, 2007

32. Schöchl H, Cotton B, Inaba K, Nienaber U, Fischer H, Voelckel W, et al: FIBTEM provides early prediction of massive transfusion in trauma. Crit Care 15:R265, 2011

33. Schwarzkopf R, Chung C, Park JJ, Walsh M, Spivak JM, Steiger D: Effects of perioperative blood product use on surgical site infection following thoracic and lumbar spinal surgery. Spine (Phila Pa 1976) 35:340-346, 2010

34. Seicean A, Alan N, Seicean S, Neuhauser D, Weil RJ: The effect of blood transfusion on short-term, perioperative outcomes in elective spine surgery. J Clin Neurosci 21:1579_ 1585,2014

35. Smart L, Mumtaz K, Scharpf D, Gray NO, Traetow D, Black $\mathrm{S}$, et al: Rotational thromboelastometry or conventional coagulation tests in liver transplantation: comparing blood loss, transfusions, and cost. Ann Hepatol 16:916-923, 2017

36. Smith JS, Klineberg E, Lafage V, Shaffrey CI, Schwab F, Lafage R, et al: Prospective multicenter assessment of perioperative and minimum 2-year postoperative complication rates associated with adult spinal deformity surgery. J Neurosurg Spine 25:1-14, 2016

37. Smith JS, Shaffrey CI, Klineberg E, Lafage V, Schwab F, Lafage R, et al: Complication rates associated with 3-column osteotomy in 82 adult spinal deformity patients: retrospective review of a prospectively collected multicenter consecutive series with 2-year follow-up. J Neurosurg Spine 27:444457, 2017

38. Smith JS, Shaffrey CI, Lafage V, Schwab F, Scheer JK, Protopsaltis T, et al: Comparison of best versus worst clinical outcomes for adult spinal deformity surgery: a retrospective review of a prospectively collected, multicenter database with 2-year follow-up. J Neurosurg Spine 23:349-359, 2015

39. Vamvakas EC, Blajchman MA: Transfusion-related mortality: the ongoing risks of allogeneic blood transfusion and the available strategies for their prevention. Blood 113:34063417, 2009

40. Veigas PV, Callum J, Rizoli S, Nascimento B, da Luz LT: 
A systematic review on the rotational thrombelastometry $\left(\right.$ ROTEM $\left.^{\circledR}\right)$ values for the diagnosis of coagulopathy, prediction and guidance of blood transfusion and prediction of mortality in trauma patients. Scand J Trauma Resusc Emerg Med 24:114, 2016

41. Wang TY, Sakamoto JT, Nayar G, Suresh V, Loriaux DB, Desai R, et al: Independent predictors of 30-day perioperative deep vein thrombosis in 1346 consecutive patients after spine surgery. World Neurosurg 84:1605-1612, 2015

42. Weber CF, Görlinger K, Meininger D, Herrmann E, Bingold T, Moritz A, et al: Point-of-care testing: a prospective, randomized clinical trial of efficacy in coagulopathic cardiac surgery patients. Anesthesiology 117:531-547, 2012

43. Weiss G, Lison S, Spannagl M, Heindl B: Expressiveness of global coagulation parameters in dilutional coagulopathy. $\mathbf{B r}$ J Anaesth 105:429-436, 2010

44. White SJW, Cheung ZB, Ye I, Phan K, Xu J, Dowdell J, et al: Risk factors for perioperative blood transfusions in adult spinal deformity surgery. World Neurosurg 115:e731-e737, 2018

45. Winter SF, Santaguida C, Wong J, Fehlings MG: Systemic and topical use of tranexamic acid in spinal surgery: a systematic review. Global Spine J 6:284-295, 2016

46. Woods BI, Rosario BL, Chen A, Waters JH, Donaldson W III, Kang J, et al: The association between perioperative allogeneic transfusion volume and postoperative infection in patients following lumbar spine surgery. J Bone Joint Surg Am 95:2105-2110, 2013

47. Xie J, Lenke LG, Li T, Si Y, Zhao Z, Wang Y, et al: Preliminary investigation of high-dose tranexamic acid for controlling intraoperative blood loss in patients undergoing spine correction surgery. Spine J 15:647-654, 2015

\section{Disclosures}

Dr. Shaffrey reports being a consultant to Medtronic, NuVasive, Zimmer Biomet, K2M, and EOS; receiving royalties from Medtronic, NuVasive, and Zimmer Biomet; and being a stock holder for NuVasive. Dr. Smith reports receiving royalties from Zimmer Biomet; being a consultant to Zimmer Biomet, Cerapedics, NuVasive, K2M, and AlloSource; receiving an honorarium from Zimmer Biomet, NuVasive, and K2M; receiving non-study-related research support from DePuy Synthes and ISSGF; and receiving fellowship support from NREF and AOSpine. Dr. Yen reports being a consultant to NuVasive.

\section{Author Contributions}

Conception and design: Buell, Naik. Acquisition of data: Buell. Analysis and interpretation of data: Buell, Naik. Drafting the article: Buell. Critically revising the article: all authors. Reviewed submitted version of manuscript: all authors. Approved the final version of the manuscript on behalf of all authors: Buell. Statistical analysis: Buell.

\section{Supplemental Information \\ Previous Presentations}

Preliminary results and earlier variations of this study were presented as an oral presentation at Eurospine 2018 (September 19-21) in Barcelona, Spain (abstract published in the 2018 abstract supplement of the European Spine Journal) and as an electronic poster presentation at the 2018 North American Spine Society Meeting (September 26-29) in Los Angeles, California (abstract published in the 2018 abstract supplement of The Spine Journal).

\section{Correspondence}

Thomas J. Buell: University of Virginia Health System, Charlottesville, VA.tjb4p@hscmail.mcc.virginia.edu. 